\title{
La biblioteca digital Mnemosine y sus colecciones: hacia una historia digital de la Edad de Plata
}

The Mnemosine Digital Library and its collections: towards a digital history of the Silver Age

\section{Dolores ROMERO LÓPEZ}

Universidad Complutense de Madrid, Departamento de Literaturas Hispánicas y Bibliografía, Madrid, España, dromero@filol.ucm.es

\begin{abstract}
Resumen
El principal objetivo de esta investigación es replantear principios historiográficos tradicionales para demostrar las posibilidades de reconstrucción y relectura de la historiografía literaria con recursos digitales. La metodología utilizada se deriva de la digitalización masiva de contenidos de parte de las bibliotecas y archivos institucionales y de su catalogación mediante metadatos que ofrecen información específica sobre los objetos digitalizados. Se presentan los primeros resultados de la investigación con un doble propósito: en primer lugar se exponen la producción, uso, difusión y almacenamiento de recursos digitales nacionales e internacionales relacionados con la relectura de la Edad de Plata. En segundo término se exponen los avances en historia digital que Mnemosine, Biblioteca Digital de La otra Edad de Plata (1868-1936) está llevando a cabo a través de la importación, configuración y reelaboración de metadatos, lo que permite la creación de colecciones específicas que se adaptan a las necesidades de los usuarios con propósitos docentes o de investigación. Esta investigación sigue en la actualidad abierta a los resultados que se obtendrán de la implementación de los metadatos para cada una de sus colecciones.
\end{abstract}

Palabras clave: Bibliotecas digitales. Biblioteca Digital Mnemosine. Literatura española de la Edad de Plata. Modelado de metadatos. Clavy.

\section{Introducción: propósito y objetivos}

El concepto Edad de Plata aplicado a la literatura surgió en 1963 de la mano de Antonio Ubieto, Juan Reglá y José María Jover quienes en su Introducción a la historia de España sostienen:

Entre 1875 a 1936 se extiende una verdadera Edad de Plata de la cultura española, durante la cual la novela, la pintura, el ensayo, la música y la lírica peninsulares van a lograr una fuerza extraordinaria como expresión de nuestra cultura nacional y un prestigio inaudito en los medios europeos [...] Este prestigio europeo de lo español

\begin{abstract}
The main objective of this research is to rethink traditional and historiographical principles to demonstrate the possibilities of reconstruction and rereading of literary historiography with digital resources. The methodology used is derived from the massive digitization of contents made by libraries and institutional archives and their metadata listing that offer specific information about digitized objects. The first results of the research are presented with a double purpose: first, the production, use, diffusion and storage of national and international digital resources related to the rereading of the Silver Age. In the second place, the advances in digital history that Mnemosine, Digital Library of the Other Silver Age (1868-1936) is carrying out through the import, configuration and processing of metadata will be presented, which allows the creation of specific collections that adapt to the needs of users for teaching or research purposes. This research is still open to the results that will be obtained from the implementation of the metadata for each of its collections.
\end{abstract}

Keywords: Digital libraries. Mnemosyne Digital Library. Spanish literature of the Silver Age. Data modelling. Clavy.

[...] no tenía precedentes desde mediados del siglo XVII (1963: 798).

La Edad de Plata surge como mímesis mermada de otro concepto historiográfico: la Edad de Oro de la literatura española en la que destacan nuestros clásicos. No hay argumentación a favor o en contra de esta nomenclatura por lo que se puede suponer que se trata de una mera intuición histórica. Igualmente, la acotación temporal de la Edad de Plata ha planteado problemas historiográficos que no es momento de abordar aquí. Basta recordar que será José Carlos Mainer quien en 1975 propone la horquilla temporal de 1902 a 1939 para acotar la Edad de Plata, fechas que han venido siendo utilizadas como 
canónicas. Francisco Abad abundará sobre la fecha de 1902 como inicio de un cambio historiográfico en la literatura de este periodo (1997: 5157). A día de hoy, Hernán Urrutia Cárdenas (1999-2000), entre otros, vuelven a abrir esa horquilla de la Edad de Plata hasta abarcar el periodo entre Repúblicas.

Será precisamente José-Carlos Mainer quien en su libro La Edad de Plata (1902-1939). Ensayo de interpretación de un proceso cultural canonice el término y lo llene de contenido sin "pretender elaborar un manual exhaustivo ni una historia social de la cultura de un periodo" (1975: 9). Efectivamente en su volumen no estaban "todos los autores, ni están todas las obras..." pero JoséCarlos Mainer logró enhebrar con eficacia documentos hemerográficos que le permitieron articular los grandes temas de la Edad de Plata: la crisis ideológica del fin de siglo, la formación de los diferentes circuitos de lectura (el burgués reformista, el popular, los regionales); la ruptura del ideal modernista; la primera etapa del vanguardismo y los nuevos vientos artísticos que se columbraron en el horizonte histórico de 1930. En fin, José Carlos Mainer ha sentado cátedra con esta terminología desde la temprana fecha de 1968 y hasta la publicación de su extenso monográfico en 2010 titulado Modernidad y nacionalismo.

De este punto de partida surge nuestra hipótesis de trabajo. Para realizar una relectura de la Edad de Plata teóricamente coherente y metodológicamente abarcable es necesario asumir -para poder superarlo- el legado histórico vertido en formato papel desde principios filológicos e historiográficos. A día de hoy, el éxito de un término como Edad de Plata se debe a la articulación que se ha venido gestando a lo largo de estos cincuenta años. En el siglo XXI el concepto Edad de Plata, al mismo tiempo que termina canonizándose en términos didácticos, inicia una doble vía de enriquecimiento teórico y metodológico. En primer lugar los estudios culturales y poscoloniales comienzan a cuestionar como eje único de articulación del periodo las mejores obras de los grandes autores, principalmente creadores masculinos. Este acercamiento teórico a la Edad de Plata viene de fuera de nuestras fronteras de la mano de Helen Graham y Jo Labanyi (1995) y Barry Jordan (2000). Incluso José Carlos Mainer en su artículo "La invención de la literatura española" (2000) habla del "canon roto". Así surge el diálogo entre Historia y Filología, por un lado, y la llamada "alteridad" de los estudios poscoloniales y la deconstrucción de la historia que son reclamadas como voces subalternas al valor canónico heredado. En segundo lugar, la digitalización masiva de contenidos a la que se van sumando progresivamente bases de datos, colecciones, museos, archivos, bibliotecas específicas (Lesk, 2005; Zhang, 2008 ; Magán y Tardón, 2014 y Solís Zara, 2016), nuevas herramientas y aplicaciones electrónicas que están dando lugar a un significativo cambio que afecta tanto a la investigación (Bembenik, 2013 y Calvo Tello, 2017) como a la didáctica en los distintos niveles (Romero, 2014a). Un paso más está pendiente de implementarse y es la posibilidad tecnológica de organizar automáticamente documentos mediante técnicas de análisis de redes (Figuerola, 2017) que podría permitir desarrollar un mapa interactivo con el conjunto de las distintas investigaciones. Esos procesos paralelos de apertura del corpus literario de la Edad de Plata y digitalización masiva de fondos históricos ha dado lugar a lo que William J. Turkle denomina el "archivo infinito" (2005-2008), un continuum de datos deslocalizados que es objeto de estudio de muchos grupos de investigación. Ante tal infinitud, surgen dos nuevos objetivos en la investigación sobre este periodo literario: 1) acotar nuevamente el campo de estudio de la Edad de Plata teniendo en cuenta los autores raros y olvidados (Romero López, 2014a), 2) crear una biblioteca digital de obras y autores de la denominada Otra Edad de Plata (Ena Bordonada, 2013 y Mnemosine) y diseñar nuevas colecciones que permitan articular la historia digital de este periodo (Colecciones Mnemosine). Alcanzar estos objetivos permitirá a docentes e investigadores localizar, seleccionar, reutilizar, enriquecer y compartir esos contenidos para proyectar su valor en la sociedad global del conocimiento.

\section{Hacia una nueva metodología: la relectura digital de la Edad de Plata}

Con el fin de articular teórica y metodológicamente lo que Anaclet Pons denomina Desorden digital (2013) se debe echar mano de la Historia digital (Melo Flórez, 2005) para demostrar cómo ha ido ganando terreno la memoria histórica en el ámbito trasnacional, sin perder un ápice de lo esencial en cualquier investigación: la búsqueda, conservación y mantenimiento del conocimiento (Berruga Sánchez, 2015). La historiografía digital se asienta en lo que Manuel Castells denomina "sociedad en red" (2017), es decir, una larga lista de enlaces que no solo sirven para dar información sino que son instrumentos que generan nuevos procesos de conocimiento en un mundo infinitamente interconectado. Hay que tener en cuenta la reciente aportación de Manfred Taller From History to Applied Science in the Humanities (2017) que presenta treinta capítulos en los que se estudian las conexiones entre la información histórica y información tecnológica y el 
trabajo de Düring (2017) sobre la lectura hermenéutica y la visualización de fuentes históricas a través de redes de datos.

Efectivamente, si buscamos la historia digital de la Edad de Plata encontramos enlaces de todo tipo. La Biblioteca Digital Hispánica facilita en búsqueda avanzada un listado de todos los libros digitalizados por campos bibliográficos, fechas, tipos de documentos y lenguas. Además su "Lista de autores en dominio público 1900-1936" pone a disposición de los investigadores 779 páginas con nombres y enlaces a autores de la Edad de Plata que han sido ya digitalizados por esa institución. El Archivo y Biblioteca de La Edad de Plata de la Residencia de Estudiantes está formado por una red de centros de instituciones culturales, públicas y privadas, especializadas en cultura española de este periodo. Destacable es el Archivo de la Junta para la Ampliación de Estudios e Investigaciones Científicas (1907-1939). En la Biblioteca Virtual Cervantes encontramos el portal Autores y Libros Raros y Olvidados, dirigido por Dolores Thion Soriano-Mollá (Universidad de Pau) que, en esta primera etapa, se centra en aquellos escritores que quedaron eclipsados por múltiples circunstancias en el Fin de Siglo, y en particular, en aquellos que contribuyeron con sus texto en Gente Nueva y el Grupo Germinal. En la Biblioteca Virtual Cervantes se encuentra el portal Editores y Editoriales Iberoamericanos siglo XIX al $X X I$, dirigido por Pura Fernández (CSIC), que presenta una amplia semblanza y memorias de editores, editoriales y colecciones de la Edad de Plata. En el ámbito de instituciones internacionales cabe destacar la biblioteca Internet Archive donde se encuentran muchos libros digitalizados de la Edad de Plata. En el portal $A$ Virtual Wunderkammer: Early Twentieth Century Erotica in Spain, dirigido por Maite Zubiaurre (2014) a través del Laboratorio de Humanidades Digitales de la Universidad de Berkeley, se hallan novelas, revistas, imágenes eróticas junto con ensayos sobre genética, pedagogía sexual, nudismo y naturalismo. Fundamentales son los proyectos particulares que se están desarrollando tanto a nivel nacional como internacional: El Archivo Digital Valle-Inclán, dirigido por Margarita Santos Zas; Online Picasso Project, dirigido desde la Sam Houston State University por Enrique Mallen y el Proyecto Aracne, coordinado por Elena Álvarez Mellado. A esto hay que sumar la labor realizada por otros investigadores particulares como José Calvo Tello (2017, grupo CLiGS) y Teresa Santa María y Elena Martínez Carro (grupo BETTE) que desde el marcado XML-TEI realizan aportaciones estilográficas. Hanno Ehrlicher (2014) y Nanette Rißler-Pipka (2017) desarrollan el portal Revistas Culturales 2.0. En el ámbito de la poesía Clara Martínez Cantón y Pablo Ruiz (2017) trabajan con corpus poéticos en el proyecto DISCO (Diachronic Corpus of Spanish Sonnets).

Lo que interesa en este momento no es la exhaustividad documental sino poner en funcionamiento la idea de que la historia digital de la Edad de Plata puede ser considerada ya hoy un hipertexto en el que encaja perfectamente la investigación desarrollada en la biblioteca digital Mnemosine.

\section{La creación de Mnemosine: Clavy, metadatos y colecciones}

Mnemosine, biblioteca digital de La otra Edad de Plata (1868-1936) surge en el seno de tres grupos de investigación vinculados a la Universidad Complutense de Madrid con el fin de crear un caso práctico que permite el uso inteligente del pasado, basado en la creación de una biblioteca rica en objetos digitales que permitan la reescritura de la historiografía literaria de la llamada Edad de Plata. En la evolución del grupo LEETHI (Literaturas Españolas y Europeas del Texto al Hipermedia) se plantea la creación de una biblioteca electrónica con libros digitalizados. Este reto reclama un campo de estudio, un periodo literario complejo, rico y diverso que se encuentra ya desarrollado en el grupo de Investigación LOEP (Literatura de la Otra Edad de Plata). Investigadores de ambos grupos, a los que se suman doctorandos de informática -pertenecientes al grupo de Investigación ILSA- y del ámbito de bibliotecas y colecciones, han sido capaces de desarrollar una biblioteca que constituye en sí misma un 'objeto de investigación' y un campo de experimentación sobre el replanteamiento de la historiografía literaria de la llamada Edad de Plata.

Mnemosine abarca un periodo comprendido entre dos fechas cruciales de la Modernidad histórica y literaria. La fecha de 1868 constituye el punto de partida ya que tras el Sexenio Democrático se desarrolla en España la novela realista, la poesía posromántica, modernista y simbolista, el teatro burgués y la zarzuela. La fecha de 1936 cercena las tendencias literarias modernas debido a la muerte, silencio o exilio de numerosos escritores.

Esta biblioteca digital surge por la necesidad de visualizar el otro canon de la Edad de Plata que fue catalogado dentro de la categoría "raros y olvidados". Como ya se ha apuntado en otro lugar (Romero López, 2014b), la primera vez que se utiliza el rótulo 'raros' como término crítico, aplicado a la literatura española es en 1863 , cuando Bartolomé José Gallardo publica su Ensayo de una biblioteca española de libros raros y curiosos. Un siglo más tarde, en 1964, Homero Serís edita Nuevo ensayo de una Biblioteca Española 
de libros raros y curiosos. Ambos libros constituyen dos pilares, que muestran la preocupación del racionalismo ilustrado por recabar para la historia de la literatura todos los escritos, no solo los más 'gloriosos'. Sin embargo en Mnemosine no se encontrarán digitalizados volúmenes anteriores a 1868. Si es cierto que el doblete "raros y curiosos" ha hecho cierta fortuna -como se puede comprobar en el despliegue de referencias que ofrece el banco de datos de la Biblioteca Nacional de España para dichos términos-, lo que interesa ahora no es tanto aquellos documentos que resultan "curiosos", sino otros que se han "olvidado". Así el concepto 'raros y olvidados' tiene en la Modernidad un significado cultural complejo referido no tanto a documentos de poca circulación o impacto, sino a aquellos que tienen una estética e ideología específicas, que no encajan en la Historia y el Canon con mayúsculas. Es Federico Carlos Sainz de Robles quien defiende el marbete 'raros y olvidados' para hablar de la literatura española del primer tercio del siglo XX. Primeramente publica su libro La novela corta española: Promoción de "El Cuento Semanal" (1901-1920) (1959) en el que se recogen algunas de las novelas olvidadas más significativas. En 1971 y 1975 vuelve sobre el tema desde una perspectiva más historicista con Raros y olvidados: (la promoción de "El Cuento Semanal") (Sainz de Robles 1959, 1971, 1975; Magnien 1986 y Î́niguez 2005). A partir de la refundación terminológica de Federico Carlos Sainz de Robles aparecen otros estudios que abundan en la necesidad de releer a autores consideramos 'menores' de este periodo. La categoría se convierte en una etiqueta, en un cajón de sastre, en el que ir acumulando lo que el canon oficial ha ido dejando fuera. Los distintos motivos que se aducen para la exclusión de autores del canon durante la Edad de Plata hay aspectos biológicos (muerte prematura del autor o nacimiento fuera de las fronteras), ideológicos (durante el franquismo se vieron excluidos del canon autores asesinados, muertos en prisión, exiliados, autoexiliados, silenciados...), genéricos (educación, sociología, estética o queer studies) y también estéticos e incluso de formato editorial.

La generalización de los procesos de digitalización en las principales instituciones culturales ha puesto a disposición de los ciudadanos en general y de los investigadores en humanidades en particular una gran cantidad de contenidos cuyo acceso era antes mucho más complicado. El acceso a estos contenidos ha estado condicionado por las características de las instituciones que han desarrollado estos proyectos (bibliotecas principalmente). Sus modelos descriptivos, sus sistemas de búsqueda y recuperación, su forma de presentar resultados y los servicios que ofrecen responden a sus necesidades y a sus posibilidades, que no coinciden necesariamente con las que el mundo de la investigación y la docencia. Por poner un ejemplo: la mera conceptualización de "Literatura de Quiosco", de "Edad de Plata" o de "Raro y Olvidado" es complicada de llevar a cabo para estas instituciones, si bien sus listados de materias muchas veces ayudan a localizar obras por criterios similares. Del mismo modo, ciertos datos que desde una perspectiva investigadora pueden ser relevantes, como el género de un autor, el precio de venta o el número de ejemplares de una tirada, difícilmente los encontraremos en un catálogo de biblioteca. Por último, determinadas herramientas o funcionalidades, como por ejemplo anexar comentarios críticos o trabajos sobre una obra, tampoco son objeto de particular interés para los Centros de Conservación, y sí pueden serlo, en cambio, desde una perspectiva académica.

Mnemosine pretende ser una biblioteca digital de acceso abierto que permita el modelado de datos de colecciones particulares (literatura de quioscos, literatura de ciencia-ficción, fotonovelas...) con el fin de apoyar la investigación y la docencia en el periodo de la Edad de Plata. El primer esbozo de Mnemosine está localizado en el servidor de la Biblioteca UCM que enlaza con las colecciones de la Biblioteca Digital HathiTrust. En la búsqueda de textos realizada en 2012 se fueron incluyendo los nombres, apellidos o seudónimos de los autores seleccionados y se localizaron un total de 2.873 textos digitalizados que se corresponden con volúmenes publicados por los autores considerados raros y olvidados. De Biblioteca Digital Hispánica, el portal de acceso a las colecciones digitales de la Biblioteca Nacional de España, proceden 2.448 obras de autores y un total de 1.017 títulos pertenecientes a mujeres escritoras.

Al margen de esta parte pública de Mnemosine, el proyecto se está desarrollando a partir de la herramienta Clavy. Clavy es un sistema de datos experimental que permite integrar fuentes externas, reconfigurar las estructuras de las colecciones. y volcar el resultado sobre otros sistemas. Clavy proporciona un sistema básico de visualización, edición y navegación. Clavy permite la importación de datos de fuentes externas (por ejemplo, HathiTrust, Biblioteca Digital Hispánica, Europeana, Library of Congress...). a través de los modelos compatibles de importación de datos bibliográficos como MARC21 y otros estándares de intercambio de datos como son XML y XLS. Se trata de un desarrollo del grupo de investigación ILSA de la Universidad Complutense que posibilita la importación, exportación y edición de registros desde múltiples formatos como 
MARC21 así como su integración en un modelo prediseñado de Mnemosine para su exportación a otros formatos compatibles como xls y $\mathrm{xml}$ (Bembenik, 2013). Como resultado, en la actualidad se han importando, a través de Clavy, a la base de datos de Mnemosine los metadatos de 5.321 objetos digitalizados procedentes de HathiTrust y de la Biblioteca Digital Hispánica. Como es lógico, los datos de estas dos fuentes se encontraban descritos en MARC21, siguiendo las reglas de catalogación bibliotecarias. Esta circunstancia ha dado lugar a un hecho esperable: en algunas ocasiones el modelo de datos de Mnemosine no ha requerido el nivel de detalle que se encuentra en el formato MARC21; y en otras, ha sido necesario incorporar datos que no se encuentran en dicho formato.

En la generación del modelo de datos de Mnemosine podemos, por tanto, distinguir tres fases: 1) El Proceso de Importación de Datos: se toma la información bibliográfica procedente de los registros bibliográficos. 2) El Proceso de Adaptación de Datos: implica cotejo, limpieza, borrado dentro de los datos importados. En esta fase se lleva a cabo el mapeo entre los elementos de MARC21 y los del modelo de datos definido para Mnemosine. Para ello, por tanto, es necesario contar ya con la definición de los elementos que compondrán dicho modelo de datos. 3) El Proceso de Enriquecimiento de Datos: En esta fase un técnico especialista en contenidos se encarga de nutrir las colecciones con los datos específicos de autores y obras. Hay que tener en cuenta que todos estos procesos deben estar guiados por el objetivo último que es favorecer la búsqueda y recuperación de la información con fines docentes y de investigación.

La importación de datos no tiene por objeto sólo el ahorro de tiempo que implicaría teclear toda esa información. Partir de la información que las bibliotecas institucionales ofrecen en sus catálogos favorece la normalización en la forma de completar los campos, especialmente en el caso del nombre de los autores. Los catálogos de autoridades de persona de las bibliotecas permiten mejorar la identificación.

Como se ha dicho, para el Proceso de Adaptación de Datos es necesario contar ya con el modelo de datos teórico de Mnemosine que se rige por principios de extensibilidad, estructuración, normalización y compatibilidad. Más allá de los principios generales seguidos, el modelo de datos que se ha implementado en Clavy hasta el momento sólo recoge dos clases (personas y obras) entre las que se establecen diferentes relaciones (es autor de; es traductor; editor, etc.) En el futuro será necesario, sin duda, ampliar el número de clases ( $\mathrm{y}$ de relaciones) para dar cabida a la descripción más precisa de sus colecciones. A la hora de crear el modelo hay que tratar de identificar los metadatos y de establecer correspondencias entre los campos y los de las diferentes bases de datos con el fin de poder incorporar esa información (Miller, 2011). Ese proceso de incorporación de información debe ser, en la medida de lo posible automático $\mathrm{y}$, en ese sentido, es necesario contar con la tecnología porque la posibilidad de importar datos de otros sitios es algo que escapa a lo meramente teórico y requiere desarrollos técnicos. Los datasets resultantes de los datos extensibles de Mnemosine se deben poder exportar a otras colecciones digitales con el fin de permitir la fluencia masiva de datos. Clavy permite la selección de datos que se deseen exportar. En un futuro próximo se integrará en Clavy un buscador avanzado, que ampliará el actual buscador simple, y que permita la búsqueda cruzada de datos. Teniendo en cuenta todo esto se han modelado dos fichas de metadatos estables en Mnemosine: Modelo de FichaAutor y Modelo de Ficha-Obra. En una biblioteca esta distinción se establece fundamentalmente con la intención de evitar errores de identificación en la autoría de las obras y para poder agrupar todas las obras de un mismo autor. La finalidad es ofrecer al usuario una ficha enciclopédica y, por tanto, especializada. La incorporación metadatos específicos en cada colección debe seguir los mismos principios teóricos del modelado general, pero también debe contener ciertas especificidades, permitidas gracias a la extensibilidad de modelado que permite Clavy.

Fruto de esta investigación teórica expuesta más arriba y de la praxis digital que permite Clavy es la creación de las primeras 10 colecciones de la biblioteca Mnemosine:

1) Mujeres Intelectuales. Esta colección integra las entradas de la biblioteca correspondientes a obras escritas por las primeras mujeres intelectuales. Se trata de obras que pertenecen a distintos géneros: poesía, novela, teatro, ensayo, y son fiel reflejo de las primeras reivindicaciones de las mujeres modernas: modernismo, librepensamiento, socialismo, feminismo, compromiso social, anarquismo, educación, periodismo, etc.

2) Literatura Infantil. Se trata de una colección que recoge obras dirigidas a un público infantil y juvenil de la Edad de Plata. Pueden hallarse primeras ediciones de obras en español e incluso cuentos traducidos de otros idiomas, lo que demuestra la dependencia de la literatura en dicho período de los gustos europeos, principalmente ingleses, franceses y alemanes. Estos relatos destacan por su valor pedagógico y moral. 
3) Madrid en la Literatura. Madrid era la ciudad moderna por excelencia durante el primer tercio del siglo XX y, por lo tanto, constituye el espacio en el que transcurre la acción en bastantes obras literarias trazando rutas emocionales que son claves para comprender los tiempos modernos.

4) Traductoras en la Edad de Plata. Esta colección es, en realidad, una sub-colección de la Mujeres Intelectuales" creada por el interés que tiene en la Edad de Plata la relación literaria con otros países europeos. Las autoras de este período fueron, además, excelentes traductoras de textos ingleses, franceses, italianos, alemanes, rusos. La traducción fue profesión de mujeres educadas en ámbitos internacionales y gracias a la traducción buena parte de dichas mujeres escritoras lograros sobrevivir en el exilio.

5) Muertos en el Conflicto. Esta colección es un hallazgo extraordinario fruto de cruce de datos que permite la biblioteca digital. En ella se rescatan del olvido todos los autores que por vejez o por motivos bélicos perdieron la vida durante la Guerra Civil. Lo interesante es además, comprobar, que muchos de estos autores han quedado libres de derechos y se puede trabajar con sus textos desde una perspectiva filológica y con los nuevos enfoques críticos de la actualidad.

6) Autores en el Exilio. Esta colección recoge los autores que sufrieron exilio a partir de 1939 y las obras de estos exiliados con el fin de geolocalizar sus itinerarios en dicho exilio.

7) Protociencia Ficción. Obras sobre los primeros textos de ciencia ficción escritos en español. Se trata de una colección pionera en España que será de obligada consulta para cualquier investigador internacional experto en el tema. Es el descubrimiento de un género que tuvo una notable difusión en la Edad de Plata.

8) Diálogos literarios. Esta colección fue creada por una investigadora que ha trabajado con el Proyecto Dialogyca sobre diálogo literario hispánico, y que se dio cuenta de que también el diálogo estaba presente en la modernidad. Es una pequeña colección que con el tiempo puede irse enriqueciendo con referencias. Supone un reto que pone a prueba Mnemosine en su función la interacción e interoperabilidad con otros grupos y bases de datos.

9) Literatura de Quioscos. Colecciones de novelas vendidas al público a precio muy económico, motivo por el cual la literatura comenzó a llegar al público económicamente menos favorecido. Son novelas sentimentales, románticas, naturalistas, pero también comprometidas, anarquistas, comunistas. Se trata de las primeras manifestaciones de la literatura de masas.
10) Carmen de Burgos. Obras de esta escritora, poeta, ensayista y periodista. Carmen de Burgos es un extraordinario exponente de escritoras ya estudiadas por investigadores del ámbito nacional e internacional. Lo más interesante de esta colección es precisamente la ficha de la autora donde se especifican todos los metadatos que sirven para definir su biografía.

\section{Resultados: la colección digital como eje historiográfico}

Cada una de las colecciones lleva un desarrollo particular que depende del investigador o los investigadores implicados y de la financiación recibida para seguir completando los metadatos. EI paso más significativo para el desarrollo de cada uno de ellas será implementar subcategorías específicas de metadatos dependiendo de los valores históricos y literarios de cada una de las colecciones. Este estudio ya se ha llevado a cabo para dos colecciones: Literatura de Quiosco y Traductoras de la Edad de Plata.

La colección Literatura de Quioscos da a conocer algunos de los libros vendidos en los quioscos durante el primer tercio del siglo XX en España. Se trata de narraciones populares, generalmente cortas, algunos de ellos escritos por mujeres, que no buscan la erudición sino el entretenimiento de las masas. Los estudios culturales han devuelto a la historiografía un necesario reposicionamiento del canon (en minúsculas) con valores de identidad incluyente (Leary \& Tangney 2003) que está permitiendo localizar, catalogar y estudiar los géneros de la literatura de masas para entender mejor el actual proceso de globalización cultural. Los quioscos "surgen en las aceras de la ciudad, como construcción de urgencia para la venta de publicaciones" (Alemán Sainz 1975:5). La literatura que allí se vende no suele tener el gran empaque que las conduce sin remedio a las bibliotecas. El interés bibliográfico por la literatura de quiosco data de los años setenta. Precursores son dos estudios: el de José María Díez Borque titulado Literatura y cultura de masas. Estudio de la novela subliteraria (1972) y Las literaturas de kiosko de Francisco Alemán Sainz (1975). Éste último introduce una clasificación internacional y ejemplos de la literatura anglosajona. José María Díez Borque analiza la literatura de quioscos como literatura de masas, tomando como referencia las publicaciones de la posguerra española. Hay que destacar la investigación de Gonzalo Santonja (1989, 1993, 2000), que desvela, analiza y edita textos de novela de quioscos de la Edad de Plata. La literatura de quioscos tuvo un enorme éxito en toda España durante el primer tercio del siglo XX. Llama la atención una cierta categorización temática según títulos de 
las colecciones. Junto a la popular colección de El Cuento Semanal (1907) de temática amplia, otras igualmente diversas son Lectura Popular (1907), Folletín Semanal (1908), Folletín (19231928), Lectura Semanal y Popular (1925-1926), Folletín Divertido (1926), Folletón (192?), Grandes Folletines (1930), Lecturas para Todos (1932). Algunas colecciones tratan de aglutinar obras de autores canónicos como Grandes Autores Contemporáneos (1929-1932). Buena parte de las colecciones aluden a la brevedad de su narración: Novela de Bolsillo (1914-1916) Novela Corta (1918-1920), Novela Chica (1924-1925), Novelita (1925) y La Novela Pequeña (1926). Otras se anuncian aludiendo al momento de su publicación: Novela del Domingo (1923) Novela del Jueves, Novela Semanal (1924), La Novela Mensual (1924); o utilizan como reclamo el momento de la lectura: Novela de Noche (1924) y Novela Nocturna (1931). No son pocas las que optan por llamar la atención sobre el tiempo de la narración: Novela de Ahora (1907), Novela de la Actualidad (1923), Novela Moderna (192?), Novela Actual (1924), Nuestra Novela (1925) y La novela nueva (1926). Los géneros que se publican en estas colecciones son tres: las menos son de poesía, varias fomentan el teatro y la mayor parte pertenecen a la narrativa corta. Teniendo en cuenta estas ideas, el diseño de los metadatos específicos de la ficha para la colección Literatura de Quioscos tendrá que tener en cuenta: título de las colecciones, el responsable intelectual de dicha colección, número dentro de la colección, número de ejemplares de la tirada, día de salida a la venta, periodicidad, puntos de venta, precio, características del formato de impresión, subgénero, personajes-tipo, conflicto, estructuras narrativas y espacio narrativo. Este modelo de datos específico permitirá búsqueda simple y avanzada de datos cruzados que dará lugar a una recuperación de la información más selectiva, mejor dirigida. La casuística podría dar lugar a datos muy interesantes desde el punto de la sociología literaria. En el momento en que se adopte el modelo de datos enlazados este esquema se sustituirá por la red de elementos y relaciones que subyacen al mismo. La búsqueda en la base de datos permitirá realizar búsquedas cruzadas complejas y pondrá de manifiesto nuevas relecturas digitales de la Edad de Plata, como por ejemplo, mujeres escritoras que publicaron literatura de quioscos y se podrá justificar las diferencias estilísticas de género y las necesidades económicas que les motivaron.

La colección Traductoras en la Edad de Plata avanza también en el mismo sentido y después de llevar a cabo un intenso estudio sobre ellas (Romero 2015, 2016) se puso en valor su producción traductora en conjunto. El número de mujeres que traducen en la Edad de Plata no es extenso pero sí significativo. Parece que las primeras traductoras modernas practican el oficio en solitario pues la labor de traducción es una labor íntima, aunque, ciertamente, las traductoras son almas inquietas, atentas al devenir cultural español y europeo, y defensoras de sus derechos. Emilia Pardo Bazán es buena prueba de ello. Condesa por propio mérito literario, antepuso el valor del trabajo personal a los honores de los títulos nobiliarios. Junto a la difusión de ideas, obras y traducciones de Balzac, Flaubert, Goncourt, Daudet, Zola, Hugo, Scribe o Legouvé, Emilia Pardo Bazán creó la revista Nuevo Teatro Crítico, en defensa de la mujer y la colección La Biblioteca de la Mujer. Además han sido traductoras: Sofía Casanova, Francisca A. de la Barella, Teresa Mañé i Miravet, Carmen de Burgos, Margarita Nelken Mausberger y su hermana Carmen Eva Nelken, Gertrude Graa, Luisi Graa, Carmen Gallardo Martín-Gamero, Carmen Abreu de Peña, Concha Méndez, Carmen Conde Abellán, Isabel Oyarzábal de Palencia, Zenobia Camprubí y Ernestina de Champourcin. Todas estas mujeres trabajan en red en torno al Lyceum, hablan como subalternas en un sistema cultural basado en patrones tradicionales masculinos y utilizan sus habilidades lingüísticas para fomentar un corpus de traducciones que apuntalan dos principios fundamentales: el valor del feminismo español y la defensa de la educación de la mujer. Los metadatos específicos que deben categorizar la colección de Traductoras de la Edad de Plata afecta por un lado a la ficha de persona que deberá contemplar: seudónimos, géneros traducidos, lenguas traducidas, lenguas conocidas, nivel de conocimiento lingüístico para cada una de las lenguas, método de adquisición, temas de los libros traducidos, actividad primaria o secundaria, actividad remunerada o no, participación en asociaciones, causas de su cese en la actividad investigadora, traducción por causa de exilio y en cada caso el país y la ciudad de exilio. Además en la ficha de obra se podrán tener en cuenta los metadatos íntegros de la obra traducida tanto en la versión original como en la traducción y que tiene que ver con el título, subtítulo, autor, formato, páginas, fecha de publicación, editorial, prologuista, ilustraciones, etc. El cruce de todos estos datos permitirá una gran riqueza en la búsqueda selectiva y despertará el interés de los investigadores no expertos por temas de investigación aún no explorados. En el momento en que se adopte el modelo de datos enlazados este esquema se sustituirá por la red de elementos y relaciones que subyacen al mismo. La búsqueda en la base de datos permitirá realizar búsquedas cruzadas complejas. Conseguir este modelo bien definido y estos datos de calidad es el primer paso para 
lograr el objetivo de facilitar la investigación y la docencia.

\section{Conclusiones}

Mediante este artículo se ha intentado demostrar que la biblioteca digital Mnemosine permite el desarrollo de competencias, destrezas y actividades que tienen que ver la reutilización de catálogos de bibliotecas y el desarrollo de nuevas herramientas digitales que permiten la reinterpretación dinámica de la historia digital de la Edad de Plata. Hemos demostrado cómo los investigadores del grupo de investigación LOEP han conseguido localizar las obras y autores raros y olvidados de la Edad de Plata con el fin de acotar el objeto de estudio. Por otro lado, la colaboración y el uso de técnicas y metodologías interdisciplinares con otros grupos de investigación ha permitido el diseño de una herramienta de importación y exportación de metadatos (Clavy) con la que se ha nutrido la biblioteca Mnemosine. Después de un proceso arduo y minucioso de revisión de todos los datos hemos creado una colección estable de datos y también de metadatos acordes al objeto de estudio. Durante el proceso de fijación de datos se detectó la necesidad de perfilar distintas colecciones específicas que suponían un nuevo planteamiento historiográfico de la Edad de Plata, fruto de la innovación digital metodológica. Cada una de estas colecciones requiere un modelado de datos específico, que debe ser desarrollado por un investigador especialista. Mnemosine es una biblioteca digital aún en estado beta que permite la experimentación con datos y metadatos de la literatura española durante la Edad de Plata. Como biblioteca reconfigurable se pone a disposición de cualquier investigación que necesite un modelado de metadatos específicos, capaces de generar búsquedas cruzadas. Mnemosine pone de manifiesto que la investigación ha de ser colaborativa e interdisciplinar a nivel de proyectos y resultados.

\section{Agradecimientos}

Este artículo se publica con el apoyo parcial de tres proyectos: "Repositorios Educativos Dinámicamente Reconfigurables en Humanidades: RedR+Human" (Ref. TIN2014-52010R, 2015-2017), "Creación, exploración y transformación de repositorios de objetos educativos en dominios especializados" (Ref. TIN2017-88092-R, 2018-2020) y eLITE-CM "Edición Literaria Electrónica (Ref. S2015/HUM-3426, 20162018).

\section{Referencias}

Abad, F. (1997). 1902 y la Edad de Plata de la cultura española. // VV.AA. (eds.). Valle-Inclán y el fin de siglo. Santiago de Compostela: Universidad de Santiago de Compostela, 1997. 51-57.
Alemán Sainz, F. (1975). Las literaturas de kiosko. Barcelona: Planeta.

Bembenik, R. (2013). Intelligent Tools for Building a Scientific Information Platform Advanced Architectures and Solutions. Berlin, Heidelberg: Springer.

Berruga Sánchez, L. (2015). La memoria nostálgica a través de Internet y de las Historias de vida. // Historia de Comunicación Social. 20:2 (2015) 391-411.

Calvo Tello, J. (2017). Estado de la digitalización de la Edad de Plata: un análisis cuantitativo. // Revista de Humanidades Digitales. 1 (2017). 76-95.

Castells, M. (2017). Internet y la sociedad red. http://www.uoc.edu/web/cat/articles/castells/casteIlsmain12.html (2017-11-28).

Colecciones Mnemosine http://repositorios.fdi.ucm.es/mnemosine/colecciones.php [Consulta: 23/11/2017]

Díez Borque, J. M., (1972). Literatura y cultura de masas: estudio de la novela subliteraria. Madrid: Al-Borak.

Düring, M. (2017). De la hermenéutica a las redes de datos: Extracción de datos y visualización de redes en fuentes históricas. // The Programming Historian. https://programminghistorian.org/es/lecciones/creando-diagramas-deredes-desde-fuentes-historicas. (2017-11-28).

Ena Bordonada, Á. ed., (2013). La otra Edad de Plata. Temas, géneros y creadores. Madrid: Ediciones Complutense.

Ehrlicher, H., Rißler-Pipka, N. (eds.) (2014). Almacenes de un tiempo en fuga. Revistas culturales en la modernidad hispánica. Aachen: Shaker.

Figuerola, C. G.; Alonso Berrocal, J. L.; Zazo Rodríguez, A. (2017). Organización automática de documentos mediante técnicas de análisis de redes. // Scire. 23:2 (juliodiciembre 2017) 25-36.

Gallardo, B. J. (1863). Ensayo de una biblioteca española de libros raros y curiosos. Madrid: Imprenta y Estereotipia de M. Rivadeneyra.

Graham, H.; Labanyi, J. (1995). Spanish Cultural Studies: an Introduction. The Struggle York: Oxford University Press.

Íñiguez Barrera, M. L. (2005). El Cuento Semanal, 19071912: análisis y estudio de una colección de novelas cortas. Granada: Grupo Editorial Universitario.

Jordan, B.; Morgan-Tamosunas, B. (eds.) (2000). Contemporary- Spanish Cultural Studies. Londres: Arnold; Nueva York: Oxford University Press.

Leary, M. R.; Tangney, J. P. (2003). Handbook of Self and Identity. Nueva York: Guilford Press.

Lesk, M. (2005). Understanding Digital Libraries. San Francisco: Morgan Kaufmann Publishers.

Magán Wals, J.A., Tardón González, E. (2014). Google libros y la digitalización masiva: la aportación de la Universidad Complutense de Madrid. // Revista General de Información y Documentación. 24: 1 9-24.

Magnien, B. (1986). Ideología y texto en El Cuento Semanal: 1907-1912. Madrid: Ediciones de la Torre.

Mainer, J. C. (1975). La Edad de Plata (1902-1939). Ensayo de interpretación de un proceso cultural. Madrid: Cátedra.

Mainer, J. C. (2000). La invención de la literatura española. // Historia, literatura y sociedad (y una coda española). Madrid: Biblioteca Nueva. 151-182.

Mainer, J. C. (2010). Historia de la literatura. Modernidad y nacionalismo. 1900-1939. Barcelona, Crítica.

Melo Flórez, J. A. (2011). Historia digital. // Historia Crítica. 43. 82-103.

Miller, S. J. (2011). Metadata for Digital Collections: A Howto-do-it Manual. Londres: Facet. 
Mnemosine. Biblioteca Digital de la Edad de Plata. http://repositorios.fdi.ucm.es/mnemosine/ (23/11/2017).

Pons, A. (2013). El desorden digital. Guía para historiadores y humanistas. Madrid: Siglo XXI.

Rißler-Pipka, N. (2017). Image and Text in Numbers: Layout Analysis for Hispanic and Spanish Modern Magazines. // Kodikologie und Paläographie im Digitalen Zeitalter 4 Codicology and Palaeography in the Digital Age 4. Schriften des Instituts für Dokumentologie und Editorik, 11. Norderstedt. 25-42.

Romero López, D. (2014a). Hacia la Smartlibrary: Mnemosine, una biblioteca digital de textos literarios raros y olvidados de la Edad de Plata (1868-1936): Fase I. // Janus Humanidades digitales: desafíos, logros y perspectivas de futuro. 411-422.

Romero López, D. (ed.), (2014b). Los márgenes de la Modernidad. Temas y creadores raros y olvidados en la Edad de Plata. Sevilla: Punto Rojo Libros.

Romero López, D. (2015). Mujeres traductoras en la Edad de Plata. Identidad moderna y Affidamento. // Hemeneus. Traducción e Interpretación. 17 (2015) 179-207.

Romero López, D. (2016). Retratos de traductoras en la Edad de Plata. Madrid: Escolar y Mayo.

Ruiz Fabo, P.; Martínez Cantón. P.; Calvo Tello, J. (2017). Diachronic Spanish Sonnet Corpus (DISCO). https://github.com/pruizf/disco. (2017-11-28).

Sainz de Robles, F. C. (1959). La novela corta española: Promoción de 'El Cuento Semanal' (1901-1920). Madrid: Aguilar.

Sainz de Robles, F. C. (1971). La promoción de "El Cuento Semanal" 1907-1925 (Un interesante e imprescindible capítulo de la historia de la novela española. Madrid: Espasa-Calpe.
Sainz de Robles, F. C. (1975). Raros y olvidados: (la promoción de 'El Cuento Semanal'). Madrid: Prensa Española.

Santonja, G. (1989). La república de los libros: el nuevo libro popular de la II República. Barcelona: Anthropos.

Santonja, G. (1993). Las obras que si escribieron algunos autores que no existen: (notas para la historia de la novela revolucionaria de quiosco en España, 1905-1939). Madrid: El Museo Universal.

Santonja, G. (2000). La insurrección literaria: la novela revolucionaria de quiosco (1905-1939). Madrid: Sial.

Serís, H. (1964). Nuevo ensayo de una Biblioteca Española de libros raros y curiosos, Valencia: Artes Gráficas Soler.

Solís Zara, S. (2016). El museo vacío. Tesis doctoral. Universidad de Sevilla.

Taller, M. (2017). From History to Applied Science in the Humanities. // Historial Social Research, 29. http://cceh.unikoeln.de/2017/10/17/hsr-supplement-29/ (2017-11-28).

Turkle, W. J. (2005). Digital History Hacks. Methodology for the Infinite Archive. // http://digitalhistoryhacks.blogspot.com.es (2017-11-28).

Ubieto, A.; Reglá, J.; Jover J. M. (1963). Introducción a la Historia de España. Barcelona: Teide.

Urrutia Cárdenas, H. (1999-2000). La Edad de Plata de la literatura española (1868-1936). // Cauce: Revista de Filología y su Didáctica 22-23 (1999-2000). 581-595.

Zhang, A. B. (2008). Creating Digital Collections: A Practical Guide. Oxford: Chandos.

Zubiaurre, M. (2014). Culturas del erotismo en España 18981939. Madrid: Cátedra.

Enviado: 2017-11-28. Segunda versión: 2018-04-25. Aceptado: 2018-05-11. 
